\title{
Hyphenation of size exclusion chromatography to native ion mobility mass spectrometry for the analytical characterization of therapeutic antibodies and related products.
}

\author{
Anthony Ehkirch ${ }^{\mathrm{a}}$, Oscar Hernandez-Alba ${ }^{\mathrm{a}}$, Olivier Colas ${ }^{\mathrm{b}}$, Alain Beck ${ }^{\mathrm{b}}$, Davy Guillarme ${ }^{\mathrm{c}}$, Sarah \\ Cianférani ${ }^{\mathrm{a}, *}$ \\ ${ }^{a}$ Laboratoire de Spectrométrie de Masse BioOrganique, Université de Strasbourg, CNRS, IPHC UMR \\ 7178, 67000 Strasbourg, France \\ ${ }^{\mathrm{b}}$ IRPF - Centre d'Immunologie Pierre-Fabre (CIPF), 74160 Saint-Julien-en-Genevois, France \\ ${ }^{c}$ School of Pharmaceutical Sciences, University of Geneva, University of Lausanne, CMU - Rue \\ Michel-Servet, 1, 1206 Geneva - Switzerland
}

Keywords: Size exclusion chromatography, native mass spectrometry, ion mobility, monoclonal antibody, antibody-drug conjugate, aggregates

\begin{abstract}
:
Mass spectrometry performed in non-denaturing conditions (native MS), and its hyphenation to ion mobility spectrometry (IM-MS), have gained interest for the qualitative and quantitative characterization of intact monoclonal antibody-related $(\mathrm{mAb})$ products. However, one main drawback is the manual sample preparation, which hampers its routine use in high throughput automated environments. Size exclusion chromatography (SEC) appears as an interesting technique to perform online buffer exchange in an automated way. We present here an exhaustive and systematic evaluation of the possibilities and versatility of SEC direct hyphenation to native MS or IM-MS (SECnativeMS/IM-MS) for the characterization of a variety of mAb-formats (IgGs, ADCs, bispecific mAbs and Fc-fusion proteins). First, online SEC-native MS allows automated sample preparation, resulting in high resolution mass spectra and improved mass accuracies $(<80 \mathrm{ppm})$ compared to manual buffer exchange procedures. When hyphenated to ion mobility, SEC-native IM-MS can deliver conformational characterization through collision cross section (CCS) measurements within few minutes without affecting mAb structures. Finally, benefits of online SEC-nativeIM-MS compared to standalone SECUV or native MS techniques are demonstrated for higher order structure characterization of $\mathrm{mAb}$ forced degraded samples. While SEC provides separation of high/low molecular weight species from the main $\mathrm{mAb}$ peak along with precise quantification of the species, native MS affords complementary unambiguous identification of SEC peaks, even when poor SEC separation is achieved. The synergic online coupling of SEC to native MS/IM-MS is envisioned to definitely push native MS approaches at the forefront of $\mathrm{mAb}$ characterization in quality-controlled environments and as multiple monitoring method.
\end{abstract}

\section{HIHGLIGHTS:}

- online SEC-nativeMS for fast and automated sample preparation for mAb analysis

- SEC-nativeIM-MS for mAb conformational characterization

- online SEC-nativeMS/IM-MS for characterization of mAb forced degraded samples 


\section{Introduction}

Monoclonal antibodies (mAbs) and their related compounds make up the largest class in human therapeutics to treat various diseases. The success of mAbs stems from their high specificity and affinity, long circulating half-lives, ability to induce immune cell effector response, and structural versatility. The development of therapeutic mAbs progress considerably, and the Food and Drug Administration (FDA) has already approved 75 therapeutic mAbs, including "naked" antibodies, radioimmunoconjugates, antibody-drug conjugates (ADC), bispecific antibodies (bsAb), Fab fragments and Fc-fusion proteins [1].

Analytical characterization of mAbs and related compounds is pivotal at all stages of compound development, from research and development phases, to process control and final batch release [2]. Straightforward analytical pipelines, often based on chromatographic, electrophoretic and mass spectrometry (MS) methods have been developed to fulfil specific normalized/certified environments requirements such as the FDA and the European Medicine Agency (EMA) [3]. Among all the MS-based techniques, native MS, and its hyphenation to ion mobility spectrometry (IM-MS) [4], have recently been successfully applied for the qualitative and quantitative characterization of intact mAb-related products in non-denaturing conditions [5-12]. However, sample preparation is still mostly manual, which hampers its routine use in high throughput and automated industrial environments. Indeed, native MS analyses have to be performed in a volatile buffer, compatible with electrospray ionization and able to maintain weak non-covalent complexes assemblies in solution [13]. Therefore, ammonium acetate buffers are classically used for native MS/IM-MS analyses [14]. Sample preparation usually consists of manual buffer exchange (also called desalting) into ammonium acetate using a variety of devices (microconcentrators, dialysis units, gel filtration devices, etc.), a procedure which is quite time consuming, labor intensive and in some cases delicate. This manual buffer exchange step thus appears as a major drawback for native MS analysis automation and throughput increase.

Size exclusion chromatography (SEC) has been suggested for many decades to be coupled to native MS/IM-MS, for performing online buffer exchange [15]. Indeed, the SEC separation is based on the differences in hydrodynamic volumes and is achieved through a column packed with particles having precise pore sizes, which makes SEC interesting for fast buffer exchange. In parallel, SEC with UV detection is routinely used in quality control laboratories for the assessment of mAb-product high molecular weight species (HMWS) [16] or for determining the content of low molecular species (LMWS), such as product fragments. Until recently, the direct coupling of SEC to MS failed, mainly because older generation of MS instruments were not sufficiently tolerant to high salt contents (even volatile salts like ammonium acetate) and the SEC columns were not enough inert (poor peak shapes were generally observed with volatile salts). Most publications report the off-line coupling of SEC to either classical denaturing MS (mostly via reversed-phase liquid chromatography coupled to MS, RPLC-MS) [17-19] or native MS [20]. In this configuration, SEC fractions are manually collected and reinjected into the MS instrument. Direct online coupling of SEC to denaturing MS, using ammonium acetate with mobile phase containing organic solvent and/or modifiers for improved chromatographic and MS performance, have been reported for proteins [21, 22] and biotherapeutics [22-29] characterization. Such experimental conditions affect protein conformation and do not allow maintaining protein aggregates. One step further has been reached with the advent of new SEC column technologies with reduced particle sizes $(<5 \mu \mathrm{m})$ and improved chemical inertness, allowing direct hyphenation of SEC to native MS. Recently, Van der Rest et al. have reported the use of online SECnative IM-MS, as a valuable technique to desalt samples and provide solution and gas-phase structural information in a single stage experiment [30]. The use of online SEC-native MS for site-specific antibody drug conjugate (3G-ADC) analysis [9] or mAb aggregates was also recently reported [31]. 
Here, we present an extensive and systematic evaluation of the possibilities to hyphenate SEC to native MS or IM-MS (further referred to as SEC-nativeMS/IM-MS) for the characterization of different mAb formats. We first evaluated the possibility and efficiency of online SEC-native MS/IM-MS for fast desalting and highlighted the versatility of the developed analytical method. Native IM-MS data obtained from off-line manual buffer exchange procedures and online SEC-native IM-MS were compared. The online SEC-native MS/IM-MS methodology was further used for 22 FDA and EMA approved antibody drugs, including IgGs, ADCs, bsAbs and Fc-fusion proteins, highlighting the method versatility. Finally, benefits of online SEC-native IM-MS compared to standalones SEC-UV or native MS techniques were demonstrated for higher order structure characterization of mAb aggregates and size-variants. 


\section{Materials and methods}

\subsection{Antibody drugs and chemicals}

Antibodies and related products were obtained as European Union pharmaceutical-grade drug product from their respective manufacturers. The NIST mAb reference Material 8671 was purchased from the National Institute Standards \& Technology (Gaithersburg, MD, USA). All chemicals were purchased from Sigma-Aldrich: ammonium acetate (A1542), cesium iodide (21004), 2-propanol (I9516). IgGZERO (A0-IZ1-010) enzyme were obtained from Genovis. All the aqueous solutions were prepared using an ultra-pure water system (Sartorius, Göttingen, Germany).

\subsection{Deglycosylation}

The deglycosylation was performed by incubating one unit of IgGZERO per microgram of ADC during 30 min at $37^{\circ} \mathrm{C}$.

\subsection{Manual Buffer exchange}

Prior to native MS, mAbs were desalted against a $150 \mathrm{mM}$ ammonium acetate solution ( $\mathrm{pH}$ 6.9) using 6 cycles of concentration/dilution on a microconcentrator (Vivaspin, 50-kD cutoff, Sartorius, Göttingen, Germany). Protein concentration was determined by UV absorbance using a NanoDrop spectrophotometer (Thermo Fisher Scientific, France).

\subsection{Preparation of forced degraded mAbs}

$100 \mu \mathrm{g}$ of trastuzumab was diluted to $1 \mathrm{mg} / \mathrm{mL}$ in $25 \mathrm{mM}$ His, $150 \mathrm{mM} \mathrm{NaCl}, 6 \%$ saccharose (w/v) $0.005 \%$ tween $80(\mathrm{v} / \mathrm{v})$ buffer at $\mathrm{pH}$ 6.5. The $\mathrm{mAb}$ was stressed by different $\mathrm{pH}$ steps as described Kukrer et al [20]. Briefly, the $\mathrm{pH}$ stress sequence consists in changing the $\mathrm{pH}$ from 6.0 to 1.0 , then from 1.0 to 10.0, from 10.0 to 1.0 and finally to adjust the $\mathrm{pH}$ to 6.0 . The stressed $\mathrm{mAb}$ was concentrated using one cycle with microconcentrator (Vivaspin, 10-kD cutoff, Sartorius, Göttingen, Germany). For NISTmAb, a $10 \mathrm{mg} / \mathrm{mL}$ solution was incubated at $40^{\circ} \mathrm{C}$ during 8 weeks.

\subsection{Size exclusion chromatography coupled to native MS and IM-MS}

An Acquity UPLC H-class system (Waters, Manchester, UK) comprising a quaternary solvent manager, a sample manager set at $10{ }^{\circ} \mathrm{C}$, a column oven and a TUV detector operating at $280 \mathrm{~nm}$ and $214 \mathrm{~nm}$ hyphenated to a Synapt G2 HDMS mass spectrometer (Waters, Manchester, UK) was used for the online SEC-native MS/IM-MS instrumentation.

Several Acquity BEH SEC columns with different nominal pore sizes, lengths and particle sizes were

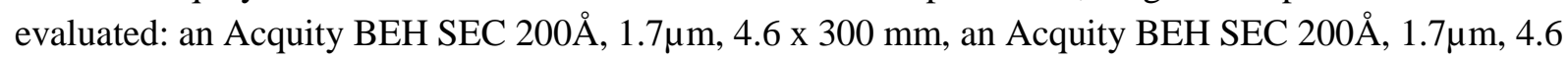

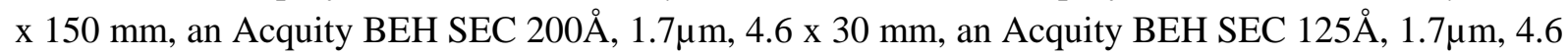
x $30 \mathrm{~mm}$, all from Waters (Waters, Manchester, UK). For benchmarking of mAb-based compounds, an Acquity BEH SEC column $(4.6 \times 150 \mathrm{~mm}, 1.7 \mu \mathrm{m}$ particle size, $200 \AA$ pore size $)$ from Waters (Manchester, UK) was used with an isocratic elution of $100 \mathrm{mM} \mathrm{NH} 4 \mathrm{OAc}(\mathrm{pH}$ 6.8) with the following flow rate gradient : $0.25 \mathrm{~mL} / \mathrm{min}$ over $4.1 \mathrm{~min}$; then, $0.10 \mathrm{~mL} / \mathrm{min}$ over $5.5 \mathrm{~min}$ and finally $0.25 \mathrm{~mL} / \mathrm{min}$ over $2.4 \mathrm{~min}$.

The Synapt G2 HDMS was operated in the positive mode with a capillary voltage of $3.0 \mathrm{kV}$. The sample cone and pressure in the interface region were set to $180 \mathrm{~V}$ and $6 \mathrm{mbar}$, respectively. Acquisitions were performed in the $\mathrm{m} / \mathrm{z}$ range $1000-8000$ for fast desalting experiments and in the $\mathrm{m} / \mathrm{z}$ range $1000-15000$ for forced degraded samples, both with a $1.5 \mathrm{~s}$ scan time. External calibration was performed using singly charged ions produced by a $2 \mathrm{~g} / \mathrm{L}$ solution of cesium iodide in 2-propanol/water $(50 / 50 \mathrm{v} / \mathrm{v})$. MS data interpretations were performed using Mass Lynx V4.1 (Waters, Manchester, UK). 
For native IM-MS experiments, the Synapt G2 HDMS instrument was carefully tuned to achieve a good compromise between ion separation and IM resolution. Low accelerating voltages were used, especially before the IM cell, to guide the ions through the mobility cell to the TOF analyzer without ion activation. The backing pressure in the interface region was $6.0 \mathrm{mbar}$, and the sampling cone was operated at either $80 \mathrm{~V}$ for benchmarking of mAb-based compounds, or $180 \mathrm{~V}$ for forced degraded samples to find out the best compromise between HMWS ion transmission and reduced assemblies dissociation. The argon flow rate in the ion trap was $5 \mathrm{~mL} / \mathrm{min}$, and the trap collision energy was set to $4 \mathrm{~V}$. Prior to IM separation, ions were thermalized in the helium cell $(130 \mathrm{~mL} / \mathrm{min})$. IM ion separation was performed in the pressurized ion mobility cell using a constant $\mathrm{N}_{2}$ flow rate of $45 \mathrm{~mL} / \mathrm{min}$. The IM wave height and velocity were $40 \mathrm{~V}$ and $923 \mathrm{~m} / \mathrm{s}$, respectively. Transfer collision energy was fixed to $2 \mathrm{~V}$ to extract the ions from the IM cell to the TOF analyzer. IM data were calibrated to perform CCS calculations using the most intense charge states of 3 external calibrants (i.e., concanavaline A, pyruvate kinase, alcohol dehydrogenase for benchmarking and glutamate dehydrogenase, pyruvate kinase, alcohol dehydrogenase for forced degraded samples) in non-denaturing conditions as described elsewhere [11, 12, 32]. IM-MS experiments were performed in triplicate under identical instrumental conditions.

\subsection{Average DAR calculation}

The average DAR value represents the sum of relative peak area for each DAR multiplied by its corresponding number of drugs [33]. It was calculated from the relative peak intensities measured either from the raw mass spectrum, by using Equation 1.

$$
D A R_{a v}=\frac{\sum_{0}^{8} k \cdot I_{k}}{\sum_{0}^{8} I_{k}}
$$

Where $k$ is the number of drugs and $I_{k}$ is the relative peak intensity of $\mathrm{DAR}_{\mathrm{k}}$. 


\section{Results and discussion}

\subsection{SEC as fast desalting method for automated native MS and IM-MS}

A first application of the hyphenation of SEC to native MS focuses on the improvement of sample preparation for native MS. Indeed, the implementation of native MS in biopharmaceutical analytical workflows is hampered by the relatively low throughput of manual and time-consuming sample preparation consisting of buffer exchange. We thus evaluated SEC, with ammonium acetate as mobile phase, as an alternative to manual desalting to reach high throughput for native MS/IM-MS analysis.

\subsubsection{Optimization of the SEC-HPLC flow rate}

We first optimized the SEC ammonium acetate flow rate using an Acquity BEH200 4.6x300mm, $1.7 \mu \mathrm{m}$ (Waters) with trastuzumab as model mAb to reach the best compromise between high MS signal intensity and efficient desalting (estimated from MS peak resolution, defined as $R_{\mathrm{FWHM}}=\mathrm{M} / \Delta \mathrm{M}_{\mathrm{FWHM}}$ ). Figure 1 (red trace) shows the SEC-native MS mass spectrum obtained with a constant ammonium acetate $(100 \mathrm{mM}, \mathrm{pH} 6.8)$ flow rate of $0.25 \mathrm{~mL} / \mathrm{min}$ classically used in LC-MS runs, which reveals a relatively low MS intensity of $3.9 \times 10^{2}$ counts for a $18 \mathrm{~min}$ run. The ammonium acetate flow rate was next reduced to $0.10 \mathrm{~mL} / \mathrm{min}$ (Fig. 1, blue trace), inducing a 5-fold increase in MS peak intensities $\left(1.8 \times 10^{3}\right.$ counts), in agreement with better ESI efficiencies at lower flow rates. Of course, the run time concomitantly increases with lower flow rates (42 min compared to $18 \mathrm{~min}$ ), which is not anymore compatible with high throughput requirements. To get a compromise between runtime and optimized native MS signals, we further optimized an ammonium acetate 3-steps flow rate gradient, in which the flow rate was initially set to $0.25 \mathrm{~mL} / \mathrm{min}$ during $4.1 \mathrm{~min}$ and sent to the waste, then it was reduced to $0.1 \mathrm{~mL} / \mathrm{min}$ during analyte elution and MS detection, and finally increased to $0.25 \mathrm{~mL} / \mathrm{min}$ again for fast non-volatile salt elution to waste.

\section{- Figure 1 -}

\subsubsection{Evaluation of different SEC columns geometries for mAb desalting}

To determine the best SEC column geometry for fast desalting of mAbs, we chose to focus on commercial BEH columns (Waters, see Material and methods), available with a variety of pore sizes and lengths but having always the same column chemistry. Online SEC-native MS analysis could be successfully achieved with any of the tested columns (see Fig. 2), but obviously longer run times were obtained with longer columns (i.e. $20 \mathrm{~min}$ for the $4.6 \times 300 \mathrm{~mm}$ ss $4 \mathrm{~min}$ for the $4.6 \times 30 \mathrm{~mm}$ using the same particle size). Slight peak tailing was observed with the longest columns (150 mm and $300 \mathrm{~mm}$, Fig. 2C and 2D) that could be mainly explained by mAb adsorption on the BEH phase. MS peak resolutions were in the same order of magnitude for all the columns ( 2300 to 2900 at m/z 5294.6, Fig. 2, right panel), with the highest resolution values obtained on the longest columns, and mass accuracies always remain lower than $40 \mathrm{ppm}$. This is in agreement with the fact that longer columns provide more efficient discrimination of low molecular weight salts and high molecular weight mAbs. As a consequence less residual salt adducts were present on the mass spectrum, leading to thinner MS peaks and subsequent increased mass accuracies. Broader MS peaks were obtained for the $125 \AA$ particle size column, as such small pore size was not adapted to $150 \mathrm{kDa}$ mAb molecules eluting in the exclusion volume of the SEC column. For high throughput purposes resulting in less than 5 min runs, the shortest columns $(30 \mathrm{~mm})$ with smaller pore size (125 or $200 \AA$ ) should be preferentially selected (see Fig. 2A and 2B). At this stage, two conclusions can be drawn: i) long columns with 20 min run times afford best chromatographic efficiencies and MS peak resolutions while ii) short columns with small pore sizes resulting in 3-5 min runs should be preferred for high throughput applications. 


\subsubsection{Comparison of online SEC-native MS and manual desalting}

We next compared the MS desalting efficiency of SEC compared to manual desalting using microconcentrators (see Material and Methods). Figure 3 presents a zoom on the 27+ charge state of trastuzumab obtained either after manual desalting (red trace) or after SEC-native MS using two different SEC columns of the same porosity but different lengths (blue and green traces). In both cases, online SEC desalting was found to be much more efficient compared to manual desalting, leading to nearly baseline resolution of trastuzumab glycoforms. A 6.7-fold increase in MS peak resolution was obtained for the fast desalting on the BEH200 4.6x30mm column vs. manual desalting, along with 20 ppm mass accuracy. Best MS peak resolutions were obviously obtained on the longest columns (BEH200 4.6x300mm), with an 8-fold increase in peak resolution leading to nearly baseline resolution of trastuzumab glycoforms along with a subsequent improved mass accuracy (7 ppm for G1F/G0F glycoform of trastuzumab by SEC-native MS on the BEH200 4.6x300mm column compared to 128 ppm by manual desalting).

\section{- Figure 3 -}

\subsubsection{Hyphenation of ion mobility to online SEC-nativeMS}

We next evaluated the possibilities to perform automated native IM-MS data acquisition. Native IMMS provides a direct size/shape measurement for global mAbs conformation assessment [5]. A good agreement was found between ${ }^{\mathrm{Tw}} \mathrm{CCS}_{\mathrm{N} 2}$ values obtained by SEC-native IM-MS and those obtained by off-line injection of manually desalted mAb formats (see Supplementary Table S1) demonstrating that SEC-native MS configuration does not affect global conformation. These results clearly demonstrate the possibility to automate IM-MS data acquisition through online SEC coupling, IM-MS data processing appears therefore as the major bottleneck towards full automation.

\subsubsection{Benchmarking of the online SEC-native MS analysis of $22 \mathrm{mAbs}$ and related compounds}

We finally aimed at benchmarking our online SEC-native MS workflow and analyzed a panel of 18 therapeutic "naked" mAbs of different types (chimeric, humanized and human), isotypes (IgG1, 2 and 4) and produced in different production systems (CHO, NSO and SP2/0) (Table 1). Based on these experiments, MS peak resolutions of the most intense charge state were higher than 2200 for all mAbs, with a distribution of values ranging from 2215 at m/z 5448.9 for panitumumab to 3542 at m/z 5347.8 for atezolizumab. Mass accuracies were always below $75 \mathrm{ppm}$, ranging from $6.7 \mathrm{ppm}$ for natalizumab and $73.8 \mathrm{ppm}$ for pembrolizumab, which is far better than the mass accuracies reported on similar QTOF instruments using the manual desalting sample preparation [11, 12]. With online SEC-native MS, nearly baseline resolution of mAb glycoforms was always achieved, bringing data obtained on Q-TOF instruments at the same level of performances, as those obtained on high resolution Orbitrap platforms $[11,12]$. No significant differences on desalting efficiencies were observed for mAbs neither as a function of their hydrophobicity nor of their pI (Table 1) [34], demonstrating the versatility of the online SEC-native MS for fast $\mathrm{mAb}$ desalting. We also analyzed some other types of mAb derivatives, including ADCs (cysteine-, lysine- or site specific ADCs) and a bispecific mAb (blinotumumab). For ADCs, drug load distributions (DLDs) along with average DARs could be unambiguously determined (Table 1, Supplementary Figure S1) from online SEC-native MS experiments (3.9 \pm 0.1 for BV, $3.4 \pm$ 0.1 for T-DM1 and 3.9 \pm 0.1 for CBW-03-106) and were in good agreement with data resulting from 
manual sample preparation [11, 12]. Altogether, our results demonstrate that the online SEC-native MS analysis can be considered as a versatile and fully automated alternative for routine native MS/IM-MS analysis, compatible with the mass accuracy, MS peak resolution and throughput requirements of biopharmaceutical industries.

- Table 1 -

\subsection{On-line SEC-IM-MS for forced degradation studies}

We next evaluated the possibilities to use online SEC-native IM-MS for the characterization of critical mAb-related HMWS impurities, such as mAb dimers, and side products derived from hinge fragmentation (LMWS) [35], that may cause immunogenic responses, have differences in pharmacokinetics or potency compared to the main product [36, 37]. As proof-of-concept, the benefits of online SEC-nativeMS/IM-MS workflow vs. SEC-UV or native MS used as standalone techniques for aggregation studies were illustrated with two representative examples: a temperature-stressed NISTmab (Fig. 4) and a pH-stressed trastuzumab sample (Fig. 5).

\subsubsection{Online SEC-native MS analysis of forced degraded NISTmAb for simultaneous LMWS and HMWS characterization}

To demonstrate the ability of our SEC-native MS methodology to simultaneously characterize HMWS and LMWS, we used a temperature-stressed NISTmAb sample to generate both aggregates and hingerelated fragments [38] (see Material and Methods). Its online SEC-native MS analysis revealed four peaks (Fig. 4A). The most intense one (peak 2) could be attributed to the monomer (148 $201 \pm 2 \mathrm{Da}$ ), while peak 1 eluting in the HMWS zone, was attributed to dimeric aggregates (296 $933 \pm 6$ Da, Fig. 4B.). The relative amount of HMWS quantified by SEC-UV was similar in both stressed and unstressed samples (around 1\%). Peaks 3 and 4, which elute after the main peak of the NISTmAb monomer, were identified as LMWS corresponding to Fc-Fab (101 $074 \pm 9$ Da) and Fab (47 $627 \pm 1$ Da) fragments. In this example, the amount of LMWS was estimated to be $4.2 \%$ from SEC-UV quantification. Comparison of stressed and unstressed NISTmAb samples shows that the fraction of HMWS stays unchanged upon stress, while an increased amount of LMWS was detected. This experiment emphasizes the ability of our SEC-native MS methods to simultaneously identify HMWS and LMWS under similar MS conditions.

\section{- Figure 4 -}

\subsubsection{Benefits of online SEC-native IM-MS analysis of pH-stressed trastuzumab vs SEC-UV or native MS as standalone techniques}

A pH stressed trastuzumab sample to generate aggregates was produced similarly to Kukrer et al. [20]. We first analyzed stressed and unstressed trastuzumab samples by native MS as standalone method, with manual desalting. As depicted in figure 5A, very similar native mass spectra were obtained, with the main ion distribution being attributed to monomeric trastuzumab and the minor one to dimers. No higher order oligomeric states could be detected neither for stressed, or for unstressed samples. As monomeric and dimeric ions are expected to have very different electrospray ionization efficiencies, it is often tricky, if not impossible, to perform relative intra-spectrum monomer/dimer ratio estimation based on standalone native MS. However, as strictly identical instrumental parameters were used in both cases, relative inter-spectra monomer/dimer ratio could be compared for stressed and unstressed conditions and estimated as $95 \%$ monomeric species and 5\% dimers. Native MS analysis with manual 
desalting thus allowed concluding that no significant differences exist, in terms of HMWS and LMWS, between stressed and unstressed trastuzumab.

We next performed online SEC-native IM-MS analysis of both samples on the longest column (Acquity BEH200 4.6x300 mm, $1.7 \mu \mathrm{m}$ ), to achieve the best possible chromatographic separation, with a $100 \mathrm{mM}$ $\mathrm{AcONH}_{4} \mathrm{pH} 6.8$ mobile phase and the flow rate gradient previously described. Figure 5B shows the SEC-UV chromatogram obtained for both stressed (red trace) and unstressed (blue trace) trastuzumab. From the sole use of SEC-UV data, a significantly higher amount of HMWS (19.0\%) was observed for $\mathrm{pH}$ stressed trastuzumab sample, which is contradictory to native MS results. However, the use of SECUV as a standalone method does not afford identification of HMWS, which clearly demonstrates the necessity and benefits of online SEC-native MS setups.

Online SEC-native IM-MS analyses were thus performed and revealed a main peak for unstressed trastuzumab, that could be attributed to monomers (148 $223 \mathrm{Da} \pm 1 \mathrm{Da}$, see Supplementary Figure S2), along with the presence of very low amounts of HMWS (below 0.5\%, as quantified by SEC-UV), which could be identified by native MS, as dimers (296 $252 \mathrm{Da} \pm 7 \mathrm{Da}$, see Supplementary Figure S2). Of note, even very low quantities of HMWS were detected by native MS with a good signal-to-noise ratio on mass spectra while no LMWS were detected. Conversely, online SEC-native IM-MS analysis of pH stressed trastuzumab revealed three peaks. The most intense peak 3 could be attributed to monomeric trastuzumab (148 $222 \pm 1 \mathrm{Da}$ ), while peaks 1 and 2 were attributed to dimeric (296 $444 \pm 4 \mathrm{Da}$ ) and trimeric (444 $667 \pm 7 \mathrm{Da}$ ) aggregates, respectively (Fig. 5C). Of note, no LMWS were detected neither for the $\mathrm{pH}$-stressed nor the unstressed trastuzumab samples. ${ }^{\mathrm{TW}} \mathrm{CCS}_{\mathrm{N} 2}$ of the different species were estimated from IM-MS data (Fig. 5D), allowing conformational characterization of each SEC separated species. ${ }^{\mathrm{TW}} \mathrm{CCS}_{\mathrm{N} 2}$ values for monomeric trastuzumab $\left(79.0 \pm 0.1 \mathrm{~nm}^{2}\right)$ were in agreement with previously published data obtained by manual injection native IM-MS analysis [12], demonstrating that SEC does not affect conformation. Conformational characterization was also obtained for dimeric $\left({ }^{\mathrm{TW}} \mathrm{CCS}_{\mathrm{N} 2}=\right.$ $\left.126.5 \pm 0.1 \mathrm{~nm}^{2}\right)$ and trimeric $\left({ }^{\mathrm{TW}} \mathrm{CCS}_{\mathrm{N} 2}=176.7 \pm 0.1 \mathrm{~nm}^{2}\right)$ HMWS. Of note, IM-MS measured ${ }^{\mathrm{TW}} \mathrm{CCS}_{\mathrm{N} 2}$ for trastuzumab oligomers were slightly higher than those predicted from the mass $\left(\mathrm{CCS}_{\text {dimer }}=108 \mathrm{~nm}^{2}\right.$, $\mathrm{CCS}_{\text {trimer }}=142 \mathrm{~nm}^{2}$ ). More interestingly, the IM-MS calculated ${ }^{\mathrm{Tw}} \mathrm{CCS}_{\mathrm{N} 2}$ values were lower than the ones calculated, considering that the CCS of a dimer should be twice (and a trimer three-times) the CCS of the monomer $\left(2 \mathrm{x}{ }^{\mathrm{TW}} \mathrm{CCS}_{\mathrm{N} 2}=158 \mathrm{~nm}^{2}, 3 \mathrm{x}{ }^{\mathrm{Tw}} \mathrm{CCS}_{\mathrm{N} 2}=237 \mathrm{~nm}^{2}\right)$, suggesting a significant conformational compaction upon $\mathrm{mAb}$ aggregation. This first example clearly highlights the benefits of online SEC-native MS/IM-MS compared to standalone techniques. Indeed, SEC provides HMWS separation which no more bias mass spectra analysis, as dimers are eluted in a different region of the chromatogram than the monomers. Consequently, SEC-based quantification of the relative HMWS was found to be more accurate than native MS-based quantitation, biased by the assumption of similar ionization efficiencies for species of very different masses. Conversely, mass identification of SECseparated species appears as a prerequisite to avoid misinterpretation due to retention time- rather than mass-based identifications. Finally, the combination of SEC-nativeMS/IM-MS is a win to win configuration as both techniques benefits from each other's advantages along with circumventing their respective drawbacks. 



\section{Conclusions}

We have presented here the potential of online SEC-native MS for the intact mass characterization of a wide range of therapeutic proteins. First, online hyphenation of SEC to native MS allows automated sample preparation (desalting), resulting in high-resolution intact protein mass spectra and subsequent accurate mass measurements $(<80 \mathrm{ppm})$. Native MS/IM-MS is now ready for high throughput routine intact mAbs analysis through its online hyphenation of SEC. We have next demonstrated the applicability of online SEC-native MS/IM-MS for forced degraded studies on several therapeutic mAbs. Having native MS directly coupled to SEC affords unambiguous identification of SEC separated species, even when poor SEC separation is achieved.

The possibility to easily hyphenate non-denaturing SEC to non-denaturing MS constitutes a real technological breakthrough, allowing automation of labor extensive manual sample preparation (i.e., buffer exchange) which is a prerequisite to succeed in native MS analysis. For protein therapeutics analysis, online SEC-native MS/IM-MS not only offers the possibility to automatize native MS analysis, but also: i) considerably improves the quality of the MS data with increased MS peak resolutions and subsequently better mass accuracies; ii) reduces the analysis time per sample from few hours (depending on the manual desalting procedure used) to few minutes without any additional sample treatment and iii) allows simultaneous quantification (through SEC-UV), identification (native MS) and conformational characterization (IM-MS) of all SEC separated species. Altogether, we believe that with online SEC-native MS, the native MS technology is ready to be integrated in intact protein high throughput analytical workflows in biopharmaceutical companies. Online SEC-native MS/IM-MS can indeed provide information on multiple critical quality attributes (i.e., glycosylation profiles, drug load distributions and average drug-to-antibody for ADCs, aggregates and fragments, etc.) within a single few minutes run, for a wide variety of mAb-related compounds through accurate intact mass measurements without any prior sample preparation.

Online SEC-native MS/IM-MS could also be of significant interest when SEC separation capabilities are exploited (e.g. for mAb aggregation studies). Indeed, the major drawback of SEC as standalone technique for aggregation studies is that it lacks MS- based accurate mass identification of the separated compounds. The direct combination of SEC to native MS is thus straightforward for unambiguous HMWS and LMWS peak assessment, affording simultaneous peak identification through accurate native MS mass measurement along with size variants quantification achieved by SEC-UV. Even if ammonium acetate is known to be not optimal for such applications [39], the possibility to have unambiguous mass-based identification online with SEC separation makes online SEC-native MS/IMMS appealing. By adding ion mobility, a direct conformational characterization of all SEC separated species is also possible within a single run. We expect that the development of new SEC column technologies will even contribute to increased throughput and improved chromatographic separation capabilities. Other perspectives of improvement rely on the development of comprehensive 2D workflows for the direct hyphenation of non-denaturing chromatography to native MS/IM-MS, as already described for online HIC hyphenation to native MS/IM-MS [40].

\section{Acknowledgments}

This work was also supported by the CNRS, the University of Strasbourg, the "Agence Nationale de la Recherche" (ANR) and the French Proteomic Infrastructure (ProFI; ANR-10-INBS-08-03). The authors thank GIS IBiSA and Région Alsace for financial support in purchasing a Synapt G2 HDMS instrument. A.E. acknowledges the «Association Nationale de la Recherche et de la Technologie » (ANRT) and Syndivia for funding his PhD fellowship. Davy Guillarme wishes to thank the Swiss National Science Foundation for support (31003A 159494). 


\section{References}

[1] P.J. Carter, G.A. Lazar, Next generation antibody drugs: pursuit of the 'high-hanging fruit', Nat Rev Drug Discov, DOI 10.1038/nrd.2017.227(2017).

[2] A. Beck, E. Wagner-Rousset, D. Ayoub, A. Van Dorsselaer, S. Sanglier-Cianferani, Characterization of therapeutic antibodies and related products, Anal Chem, 85 (2013) 715-736.

[3] C.N. Ferguson, A.C. Gucinski-Ruth, Evaluation of Ion Mobility-Mass Spectrometry for Comparative Analysis of Monoclonal Antibodies, J Am Soc Mass Spectrom, 27 (2016) 822-833.

[4] S. Rogstad, A. Faustino, A. Ruth, D. Keire, M. Boyne, J. Park, A Retrospective Evaluation of the Use of Mass Spectrometry in FDA Biologics License Applications, J Am Soc Mass Spectrom, 28 (2017) 786794.

[5] A. Beck, S. Sanglier-Cianferani, A. Van Dorsselaer, Biosimilar, biobetter, and next generation antibody characterization by mass spectrometry, Anal Chem, 84 (2012) 4637-4646.

[6] S. Rosati, N.J. Thompson, A. Barendregt, L.J. Hendriks, A.B. Bakker, J. de Kruif, M. Throsby, E. van Duijn, A.J. Heck, Qualitative and semiquantitative analysis of composite mixtures of antibodies by native mass spectrometry, Anal Chem, 84 (2012) 7227-7232.

[7] S. Rosati, Y. Yang, A. Barendregt, A.J. Heck, Detailed mass analysis of structural heterogeneity in monoclonal antibodies using native mass spectrometry, Nat Protoc, 9 (2014) 967-976.

[8] N.J. Thompson, L.J. Hendriks, J. de Kruif, M. Throsby, A.J. Heck, Complex mixtures of antibodies generated from a single production qualitatively and quantitatively evaluated by native Orbitrap mass spectrometry, MAbs, 6 (2014) 197-203.

[9] T. Botzanowski, S. Erb, O. Hernandez-Alba, A. Ehkirch, O. Colas, E. Wagner-Rousset, D. Rabuka, A. Beck, P.M. Drake, S. Cianferani, Insights from native mass spectrometry approaches for top- and middle- level characterization of site-specific antibody-drug conjugates, Mabs, 9 (2017) 801-811.

[10] F. Debaene, E. Wagner-Rousset, O. Colas, D. Ayoub, N. Corvaia, A. Van Dorsselaer, A. Beck, S. Cianferani, Time resolved native ion-mobility mass spectrometry to monitor dynamics of IgG4 Fab arm exchange and "bispecific" monoclonal antibody formation, Anal Chem, 85 (2013) 9785-9792.

[11] F. Debaene, A. Boeuf, E. Wagner-Rousset, O. Colas, D. Ayoub, N. Corvaia, A. Van Dorsselaer, A. Beck, S. Cianferani, Innovative native MS methodologies for antibody drug conjugate characterization: High resolution native MS and IM-MS for average DAR and DAR distribution assessment, Anal Chem, 86 (2014) 10674-10683.

[12] J. Marcoux, T. Champion, O. Colas, E. Wagner-Rousset, N. Corvaia, A. Van Dorsselaer, A. Beck, S. Cianferani, Native mass spectrometry and ion mobility characterization of trastuzumab emtansine, a lysine-linked antibody drug conjugate, Protein Sci, 24 (2015) 1210-1223.

[13] E. van Duijn, Current limitations in native mass spectrometry based structural biology, J Am Soc Mass Spectrom, 21 (2010) 971-978.

[14] J. Marcoux, S. Cianferani, Towards integrative structural mass spectrometry: Benefits from hybrid approaches, Methods, 89 (2015) 4-12.

[15] G.M. Waitt, R. Xu, G.B. Wisely, J.D. Williams, Automated in-line gel filtration for native state mass spectrometry, J Am Soc Mass Spectrom, 19 (2008) 239-245.

[16] D. Lowe, K. Dudgeon, R. Rouet, P. Schofield, L. Jermutus, D. Christ, Aggregation, stability, and formulation of human antibody therapeutics, Adv Protein Chem Struct Biol, 84 (2011) 41-61.

[17] W. Cai, T. Tucholski, B. Chen, A.J. Alpert, S. Mcllwain, T. Kohmoto, S. Jin, Y. Ge, Top-Down Proteomics of Large Proteins up to 223 kDa Enabled by Serial Size Exclusion Chromatography Strategy, Anal Chem, 89 (2017) 5467-5475.

[18] J.A. Pavon, X. Li, S. Chico, U. Kishnani, S. Soundararajan, J. Cheung, H. Li, D. Richardson, M. Shameem, X. Yang, Analysis of monoclonal antibody oxidation by simple mixed mode chromatography, J Chromatogr A, 1431 (2016) 154-165.

[19] S. Zheng, D. Qiu, M. Adams, J. Li, R.V. Mantri, R. Gandhi, Investigating the Degradation Behaviors of a Therapeutic Monoclonal Antibody Associated with pH and Buffer Species, AAPS PharmSciTech, 18 (2017) 42-48. 
[20] B. Kukrer, V. Filipe, E. van Duijn, P.T. Kasper, R.J. Vreeken, A.J. Heck, W. Jiskoot, Mass spectrometric analysis of intact human monoclonal antibody aggregates fractionated by size-exclusion chromatography, Pharm Res, 27 (2010) 2197-2204.

[21] M.T. Smoluch, P. Mak, J.P. Chervet, G. Hohne, J. Silberring, Size-exclusion chromatography performed in capillaries. Studies by liquid chromatography-mass spectrometry, J Chromatogr B Biomed Sci Appl, 726 (1999) 37-43.

[22] M.L. Shen, L.M. Benson, K.L. Johnson, J.J. Lipsky, S. Naylor, Effect of enzyme inhibitors on protein quaternary structure determined by on-line size exclusion chromatography-microelectrospray ionization mass spectrometry, J Am Soc Mass Spectrom, 12 (2001) 97-104.

[23] A.C. Lazar, L. Wang, W.A. Blattler, G. Amphlett, J.M. Lambert, W. Zhang, Analysis of the composition of immunoconjugates using size-exclusion chromatography coupled to mass spectrometry, Rapid Commun Mass Spectrom, 19 (2005) 1806-1814.

[24] L.J. Brady, J. Valliere-Douglass, T. Martinez, A. Balland, Molecular mass analysis of antibodies by on-line SEC-MS, J Am Soc Mass Spectrom, 19 (2008) 502-509.

[25] A. Wakankar, Y. Chen, Y. Gokarn, F.S. Jacobson, Analytical methods for physicochemical characterization of antibody drug conjugates, MAbs, 3 (2011) 161-172.

[26] B. Bobaly, S. Fleury-Souverain, A. Beck, J.L. Veuthey, D. Guillarme, S. Fekete, Current possibilities of liquid chromatography for the characterization of antibody-drug conjugates, J Pharm Biomed Anal, 147 (2018) 493-505.

[27] C.F. Xu, L. Zang, A. Weiskopf, Size-exclusion chromatography-mass spectrometry with mnitrobenzyl alcohol as post-column additive for direct characterization of size variants of monoclonal antibodies, J Chromatogr B Analyt Technol Biomed Life Sci, 960 (2014) 230-238.

[28] J.F. Valliere-Douglass, S.M. Hengel, L.Y. Pan, Approaches to Interchain Cysteine-Linked ADC Characterization by Mass Spectrometry, Mol Pharm, 12 (2015) 1774-1783.

[29] J.F. Valliere-Douglass, W.A. McFee, O. Salas-Solano, Native intact mass determination of antibodies conjugated with monomethyl Auristatin $\mathrm{E}$ and $\mathrm{F}$ at interchain cysteine residues, Anal Chem, 84 (2012) 2843-2849.

[30] G. Van der Rest, F. Halgand, Size Exclusion Chromatography-Ion Mobility-Mass Spectrometry Coupling: a Step Toward Structural Biology, J Am Soc Mass Spectrom, 28 (2017) 2519-2522.

[31] M. Haberger, M. Leiss, A.K. Heidenreich, O. Pester, G. Hafenmair, M. Hook, L. Bonnington, H. Wegele, M. Haindl, D. Reusch, P. Bulau, Rapid characterization of biotherapeutic proteins by sizeexclusion chromatography coupled to native mass spectrometry, MAbs, 8 (2016) 331-339.

[32] M.F. Bush, Z. Hall, K. Giles, J. Hoyes, C.V. Robinson, B.T. Ruotolo, Collision cross sections of proteins and their complexes: a calibration framework and database for gas-phase structural biology, Anal Chem, 82 (2010) 9557-9565.

[33] A. Beck, G. Terral, F. Debaene, E. Wagner-Rousset, J. Marcoux, M.C. Janin-Bussat, O. Colas, A. Van Dorsselaer, S. Cianferani, Cutting-edge mass spectrometry methods for the multi-level structural characterization of antibody-drug conjugates, Expert Rev Proteomics, 13 (2016) 157-183.

[34] A. Goyon, M. Excoffier, M.C. Janin-Bussat, B. Bobaly, S. Fekete, D. Guillarme, A. Beck, Determination of isoelectric points and relative charge variants of 23 therapeutic monoclonal antibodies, J Chromatogr B Analyt Technol Biomed Life Sci, 1065-1066 (2017) 119-128.

[35] O.O. Dada, R. Rao, N. Jones, N. Jaya, O. Salas-Solano, Comparison of SEC and CE-SDS methods for monitoring hinge fragmentation in IgG1 monoclonal antibodies, J Pharm Biomed Anal, 145 (2017) 9197.

[36] A.S. De Groot, D.W. Scott, Immunogenicity of protein therapeutics, Trends Immunol, 28 (2007) 482-490.

[37] A.S. Rosenberg, Effects of protein aggregates: an immunologic perspective, AAPS J, 8 (2006) E501507.

[38] J. Vlasak, R. lonescu, Fragmentation of monoclonal antibodies, MAbs, 3 (2011) 253-263.

[39] A. Goyon, V. D'Atri, O. Colas, S. Fekete, A. Beck, D. Guillarme, Characterization of 30 therapeutic antibodies and related products by size exclusion chromatography: Feasibility assessment for future 
mass spectrometry hyphenation, J Chromatogr B Analyt Technol Biomed Life Sci, 1065-1066 (2017) 3543.

[40] A. Ehkirch, V. D'Atri, F. Rouviere, O. Hernandez-Alba, A. Goyon, O. Colas, M. Sarrut, A. Beck, D. Guillarme, S. Heinisch, S. Cianferani, An Online Four-Dimensional HICxSEC-IMxMS Methodology for Proof-of-Concept Characterization of Antibody Drug Conjugates, Anal Chem, DOI 10.1021/acs.analchem.7b02110(2018).

[41] B.T. Ruotolo, J.L. Benesch, A.M. Sandercock, S.J. Hyung, C.V. Robinson, lon mobility-mass spectrometry analysis of large protein complexes, Nat Protoc, 3 (2008) 1139-1152. 


\section{Figure captions:}

Fig. 1. Native mass spectra of trastuzumab analyzed by online SEC-native MS with a constant flow rate of $0.25 \mathrm{ml} / \mathrm{min}$ (red trace) and a flow rate of $0.1 \mathrm{~mL} / \mathrm{min}$ during the elution (blue trace).

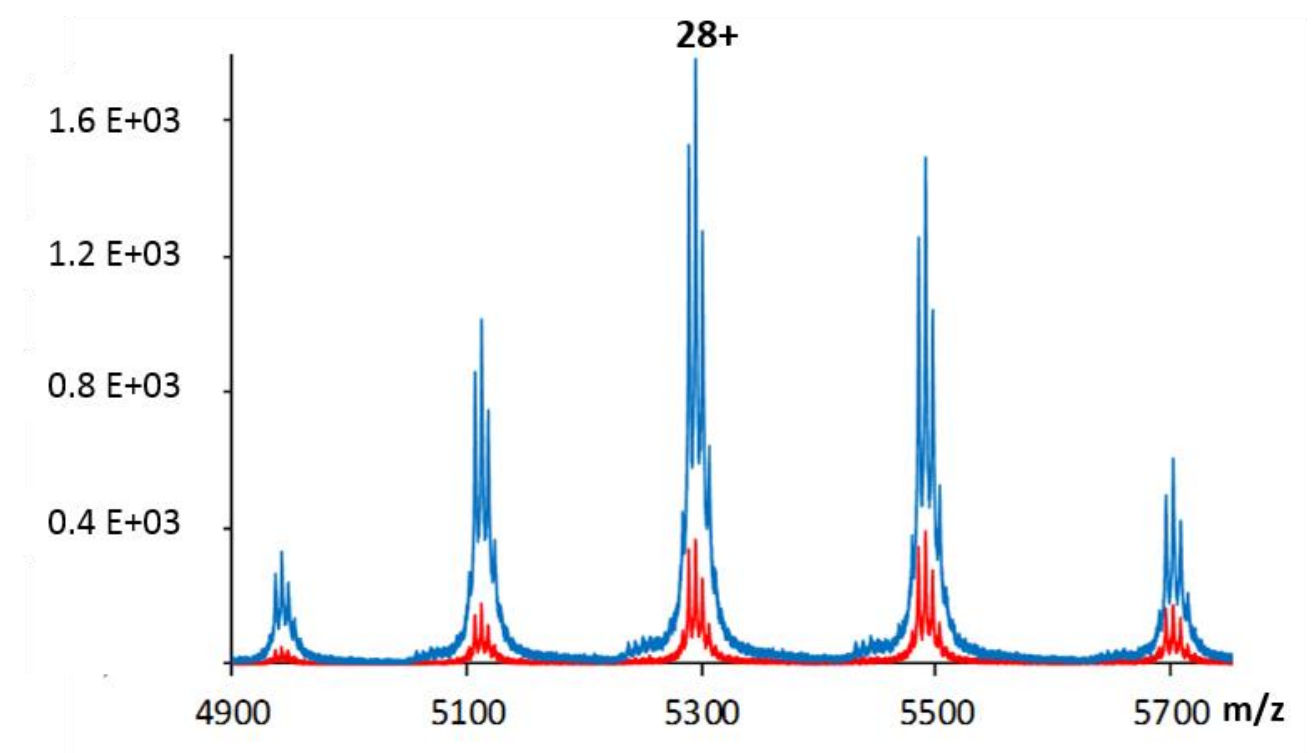

Fig. 2. Evaluation of different SEC columns for fast mAb desalting on trastuzumab (A-D). Left panels: SEC chromatograms obtained with corresponding flow rate gradients. Right panels: zoom on the 28+ charge state of trastuzumab in native MS conditions. MS peak resolution are given for $\mathrm{m} / \mathrm{z} 5294$.6. Mass accuracies are provided for the G1F/G0F glycoform.

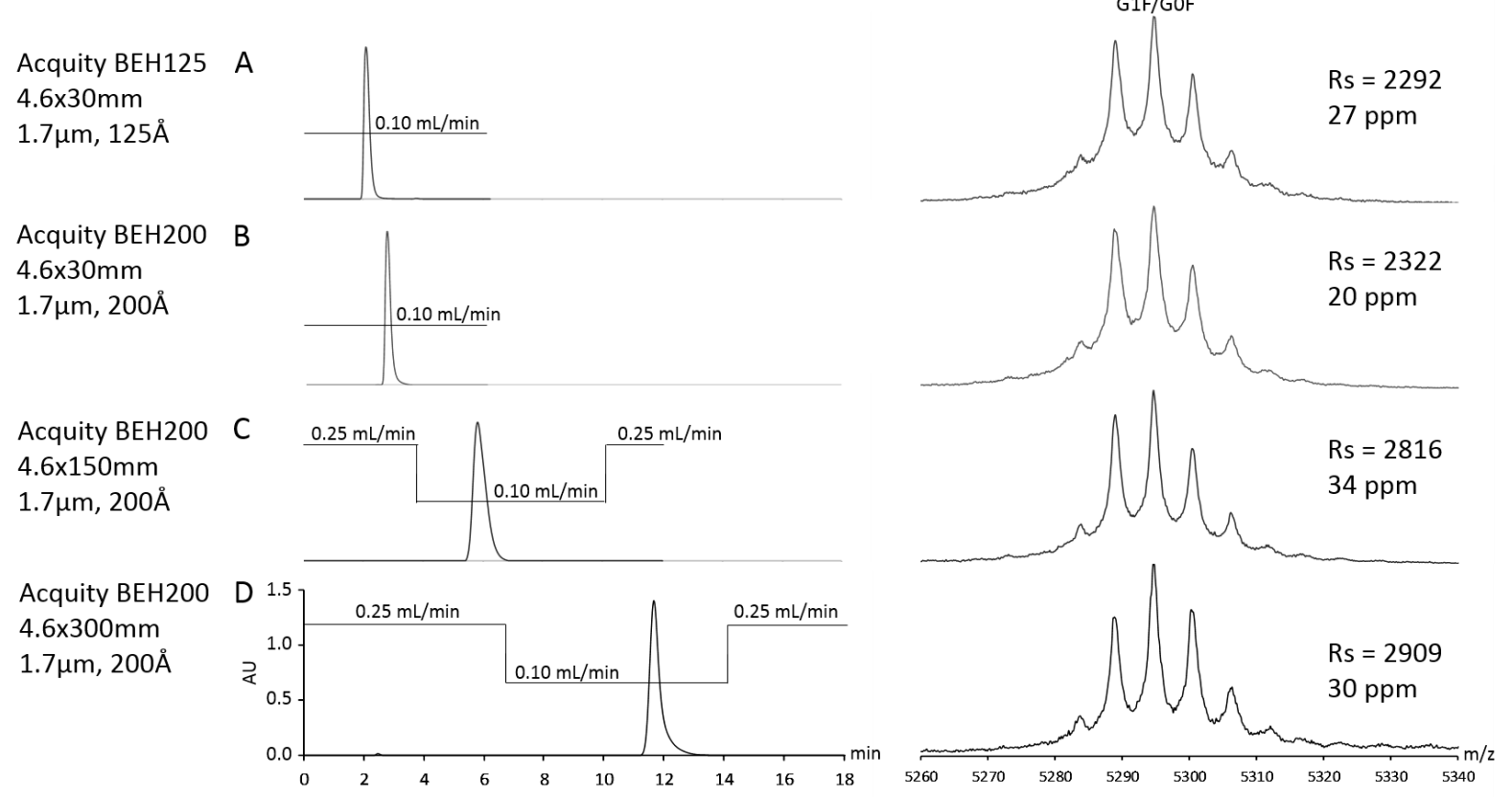

Fig. 3. Comparison of manual and SEC desalting efficiencies. Zoom on the 27+ charge state of trastuzumab manually desalted (red trace) and analyzed by SEC-native MS with an Acquity BEH200 
4.6x30mm (green trace) and with an Acquity BEH200 4.6x300mm (blue trace). MS peak resolutions were given for $\mathrm{m} / \mathrm{z} 5490.7$ while mass accuracies are provided for the G1F/G0F glycoform of trastuzumab.

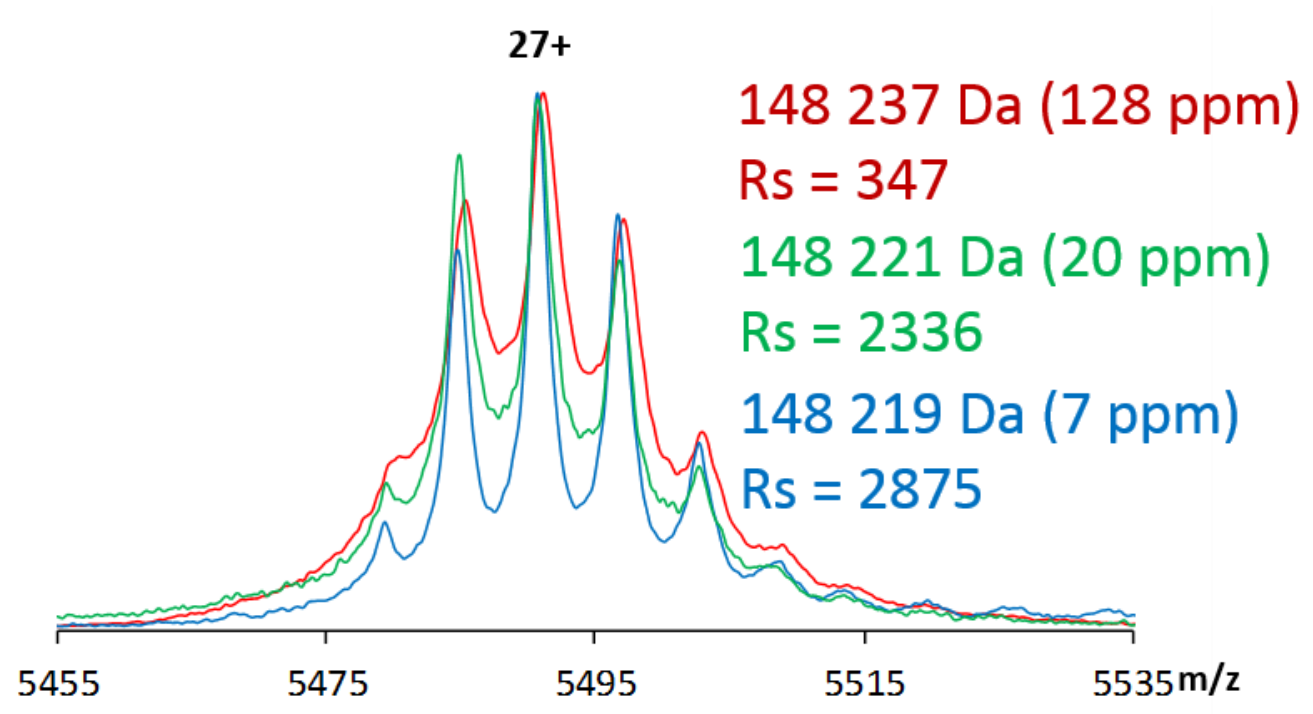

Fig. 4. Online SEC-native MS analysis of temperature-stressed NISTmAb sample. (A) Overlaid SEC chromatogram of stressed (red trace) and unstressed (blue trace) NISTmAb. (B) Native mass spectra of each individual SEC separated peak for the temperature-stressed NISTmAb sample.

A

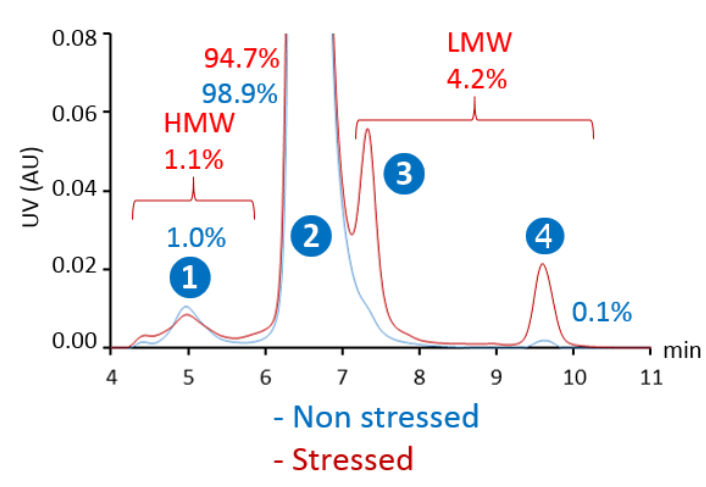

B Dimer

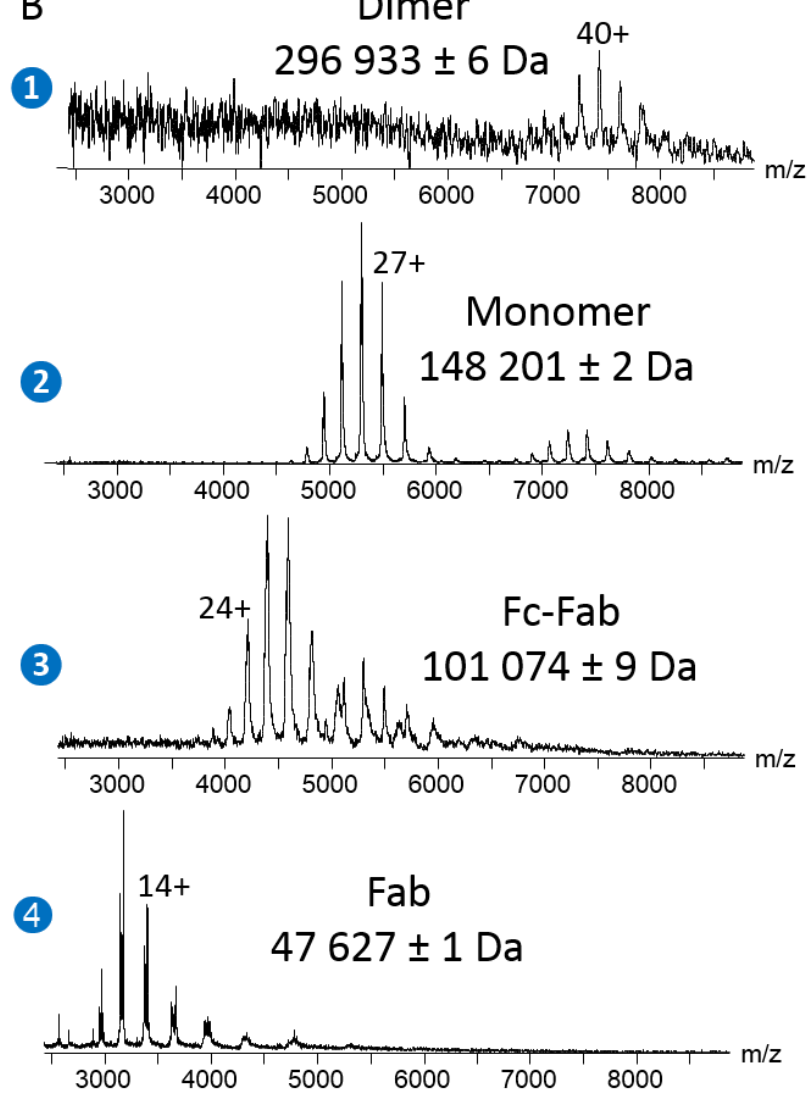

Fig. 5. Online SEC-native MS/IM-MS analysis of pH-stressed trastuzumab. (A) Native mass spectra of stressed and unstressed trastuzumab with manual desalting. (B) Overlaid SEC chromatograms of 
stressed (red trace) and unstressed (blue trace) trastuzumab. (C) Native mass spectra of each individual SEC separated peak for the $\mathrm{pH}$ stressed sample. (D) Ion mobility arrival time distributions (ATDs) of monomeric, dimeric and trimeric species along with their collision cross section (CCS) (28+ charge state for the monomer, $40+$ charge state for the dimer and $52+$ charge state of the trimer). Solid lines represent theoretical CCS calculated through the equation CCS $=2.435^{*} \mathrm{M}^{2 / 3}$ for spherical proteins [41].
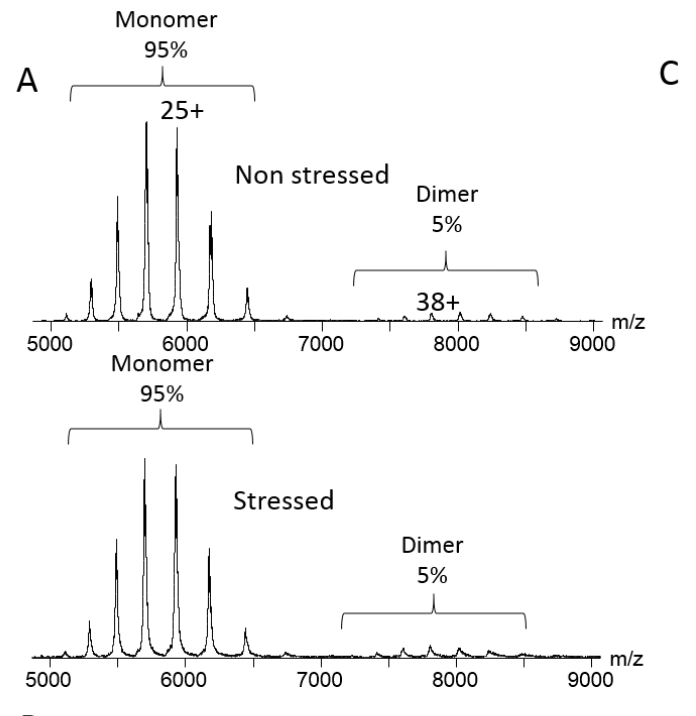

B
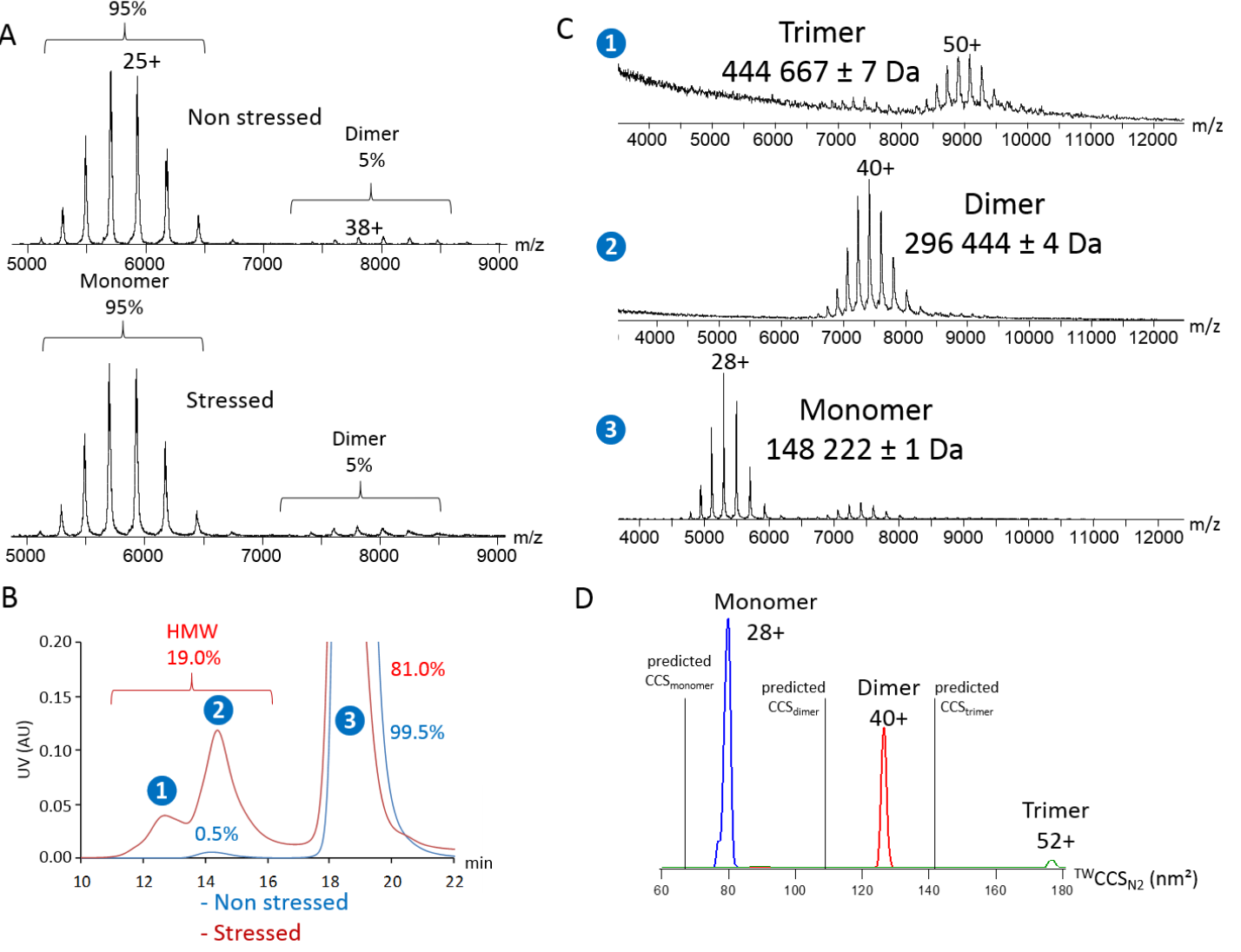

D

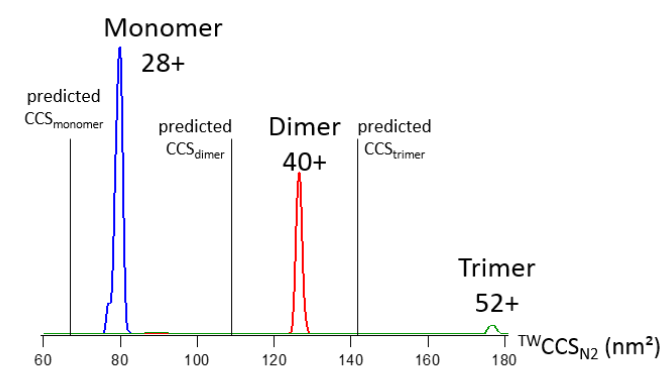




\section{Table legend}

Table 1. Benchmarking of SEC-native MS analysis of 22 therapeutic proteins. Molecular weights are reported for the G1F/G0F glycoforms, MS peak resolutions were obtained from the 27+ charge state.

\begin{tabular}{|c|c|c|c|c|c|c|c|c|}
\hline & & Isotype & $\mathrm{pl}$ & $\begin{array}{l}\text { HIC apparent } \\
\text { retention factor }\end{array}$ & $\begin{array}{c}\text { Theoretical mass } \\
\text { based on } \\
\text { G1F/GOF (Da) }\end{array}$ & $\begin{array}{l}\text { Experimental } \\
\text { mass based on } \\
\text { G1F/GOF (Da) }\end{array}$ & $\begin{array}{c}\text { Mass } \\
\text { accuracy } \\
(\text { ppm) }\end{array}$ & $\begin{array}{c}\text { Rs on } 27+ \\
\text { charge state }\end{array}$ \\
\hline \multirow{18}{*}{ mAbs } & adalimumab & $\lg G 1$ & 8.9 & 1.1 & 148244 & 148238 & 40.5 & 2842.3 \\
\hline & atezolizumab & $\lg G 1$ & 8.8 & 6.0 & $\begin{array}{c}144356 \\
\text { (a-glycosylated) }\end{array}$ & 144362 & 41.6 & 3541.6 \\
\hline & bevacizumab & $\lg G 1$ & 8.3 & 4.6 & 149361 & 149365 & 26.8 & 2908.9 \\
\hline & brentuximab B & $\lg G 1$ & & & 148364 & 148368 & 29.3 & 2376.8 \\
\hline & denosumab & $\lg \mathrm{g} 2$ & 8.8 & 1.1 & 147515 & 147519 & 27.1 & 3133.1 \\
\hline & eculizumab & $\lg G 2 / 4$ & 6.1 & 1.7 & 148033 & 148035 & 13.5 & 3043.3 \\
\hline & elotuzumab & $\lg G 1$ & 8.3 & 2.6 & 148088 & 148096 & 50.2 & 2364.6 \\
\hline & infliximab & $\lg G 1$ & 7.6 & 3.1 & 148675 & 148681 & 40.4 & 2413.1 \\
\hline & ipilimumab & $\lg G 1$ & 9.2 & 4.3 & 148154 & 148152 & 13.5 & 3168.9 \\
\hline & natalizumab & $\lg G 4$ & 7.8 & 2.2 & 148973 & 148974 & 6.7 & 3470.6 \\
\hline & NISTmab & $\lg G 1$ & 9.0 & 1.7 & 148201 & 148198 & 20.2 & 2967.5 \\
\hline & nivolumab & $\operatorname{lgG} 4$ & 8.0 & 2.3 & 146384 & 146390 & 44.4 & 2928.2 \\
\hline & ofatumumab & $\lg G 1$ & 8.9 & 2.1 & 148859 & 148867 & 55.5 & 3243.9 \\
\hline & panitumumab & $\lg G 2$ & 6.8 & 1.8 & 147087 & 147094 & 47.6 & 2215.0 \\
\hline & pembrolizumab & $\lg G 4$ & 7.6 & 5.5 & 149051 & 149062 & 73.8 & 2664.6 \\
\hline & ramucirumab & $\lg G 1$ & 8.9 & 1.6 & 146592 & 146587 & 34.1 & 2505.1 \\
\hline & rituximab & $\lg G 1$ & 9.4 & 3.8 & 147237 & 147239 & 13.6 & 2525.1 \\
\hline & trastuzumab & $\lg G 1$ & 9.0 & 1.9 & 148218 & 148219 & 6.7 & 2874.7 \\
\hline $\mathrm{bAb}$ & blinatumomab & scFv-scFv & & & 54087 & 54085 & 37.0 & $3005.9(15+)$ \\
\hline \multirow{15}{*}{ ADCs } & \multirow{5}{*}{$\begin{array}{c}\text { brentuximab } \\
\text { vedotin } \\
\text { (deglycosylated) }\end{array}$} & \multirow{5}{*}{$\lg G 1$} & & \multirow{5}{*}{$\begin{array}{c}\text { Average DAR } \\
3.9\end{array}$} & D0 $14588814.4 \%$ & 145899 & 75.4 & \\
\hline & & & & & D2 $14852323.5 \%$ & 148525 & 13.5 & \\
\hline & & & & & D4 $15115828.8 \%$ & 151166 & 52.9 & 2014.4 \\
\hline & & & & & D6 $15379420.7 \%$ & 153801 & 45.5 & \\
\hline & & & & & D8 $15642912.6 \%$ & 156441 & 76.7 & \\
\hline & \multirow{8}{*}{$\begin{array}{l}\text { trastuzumab } \\
\text { emtansine } \\
\text { (deglycosylated) }\end{array}$} & \multirow{8}{*}{$\operatorname{lgG1}$} & & \multirow{8}{*}{$\begin{array}{c}\text { Average DAR } \\
3.4\end{array}$} & D0 $1458647.9 \%$ & 145858 & 41.1 & \\
\hline & & & & & D1 146822 11.0\% & 146819 & 20.4 & \\
\hline & & & & & D2 147779 15.8\% & 147782 & 20.3 & \\
\hline & & & & & D3 $14873718.5 \%$ & 148739 & 13.5 & 2103.0 \\
\hline & & & & & D4 149694 16.9\% & 149691 & 20.0 & \\
\hline & & & & & D5 $15065213.4 \%$ & 150651 & 6.6 & \\
\hline & & & & & D6 151609 9.1\% & 151609 & 6.6 & \\
\hline & & & & & D7 $1525677.4 \%$ & 152556 & 72.1 & \\
\hline & \multirow{2}{*}{$\begin{array}{c}\text { CBW-03-106 } \\
\text { (deglycosylated) }\end{array}$} & \multirow{2}{*}{$\lg G 1$} & & \multirow{2}{*}{$\begin{array}{c}\text { Average DAR } \\
3.9\end{array}$} & D3 $1515537 \%$ & 151546 & 46.2 & \\
\hline & & & & & D4 152771 93\% & 152773 & 13.1 & 1849.8 \\
\hline
\end{tabular}




\section{SUPPORTING INFORMATION}

Hyphenation of size exclusion chromatography to native ion mobility mass spectrometry for the analytical characterization of therapeutic antibodies and related products.

Anthony Ehkirch ${ }^{\mathrm{a}}$, Oscar Hernandez-Alba ${ }^{\mathrm{a}}$, Olivier Colas ${ }^{\mathrm{b}}$, Alain Beck ${ }^{\mathrm{b}}$, Davy Guillarme ${ }^{\mathrm{c}}$, Sarah

Cianférani $\mathrm{a}^{\mathrm{a},}$

${ }^{\text {a }}$ Laboratoire de Spectrométrie de Masse BioOrganique, Université de Strasbourg, CNRS, IPHC UMR 7178, 67000 Strasbourg, France

${ }^{\mathrm{b}}$ IRPF - Centre d'Immunologie Pierre-Fabre (CIPF), 74160 Saint-Julien-en-Genevois, France

${ }^{c}$ School of Pharmaceutical Sciences, University of Geneva, University of Lausanne, CMU - Rue Michel-Servet, 1, 1206 Geneva - Switzerland

\section{TABLE OF CONTENTS:}

- Supplementary Table S1: Comparison of ${ }^{\mathrm{TW}} \mathrm{CCS}_{\mathrm{N} 2}$ values experimentally obtained by SECnative IM-MS and off-line injection of manually desalted mAb formats, for 18 therapeutic mAbs and ADCs.

- Supplementary Figure S1: Deconvoluted mass spectra of brentuximab vedotin (A), trastuzumab emtansine (B) and CBW-03-106 site specific ADC (C) obtained from online SEC-native MS.

- Supplementary Figure S2: Online SEC-native MS analysis of unstressed trastuzumab. (A) SEC chromatogram of unstressed trastuzumab. (B) Native mass spectra of each individual SEC peak. 


\begin{tabular}{|c|c|c|c|c|}
\hline & & $\begin{array}{c}\text { SEC-IM-MS } \\
\text { experimental } \\
\mathrm{TW}_{\mathrm{CCS}_{\mathrm{N} 2}\left(\mathrm{~nm}^{2}\right)} \\
26+\end{array}$ & $\begin{array}{c}\text { Manual } \\
\text { injection } \\
\mathrm{TW}_{\mathrm{CCS}_{\mathrm{N} 2}\left(\mathrm{~nm}^{2}\right)} \\
26+\end{array}$ & $\begin{array}{l}\text { Predicted } \\
\operatorname{CCS}\left(\mathrm{nm}^{2}\right)\end{array}$ \\
\hline \multirow{16}{*}{ mAbs } & adalimumab & $78.78 \pm 0.10$ & & 68.24 \\
\hline & atezolizumab & $78.79 \pm 0.10$ & & 67.01 \\
\hline & bevacizumab & $78.97 \pm 0.10$ & & 68.53 \\
\hline & brentuximab B & $79.03 \pm 0.10$ & & 68.20 \\
\hline & denosumab & $79.86 \pm 0.10$ & & 67.94 \\
\hline & elotuzumab & $79.33 \pm 0.10$ & & 68.17 \\
\hline & ipilimumab & $79.45 \pm 0.10$ & & 68.25 \\
\hline & natalizumab & $79.15 \pm 0.10$ & & 68.53 \\
\hline & nistmab & $78.42 \pm 0.10$ & $78.49 \pm 0.10$ & 67.48 \\
\hline & nivolumab & $78.16 \pm 0.10$ & & 67.71 \\
\hline & ofatumumab & $79.27 \pm 0.10$ & & 68.52 \\
\hline & panitumumab & $79.03 \pm 0.10$ & & 67.92 \\
\hline & pembrolizumab & $79.09 \pm 0.10$ & & 68.50 \\
\hline & ramucirumab & $78.99 \pm 0.10$ & & 67.83 \\
\hline & rituximab & $78.78 \pm 0.10$ & & 68.02 \\
\hline & trastuzumab & $78.78 \pm 0.10$ & & 68.21 \\
\hline \multirow{2}{*}{ ADCs } & $\begin{array}{c}\text { brentuximab } \\
\text { vedotin } \\
\text { (deglycosylated) }\end{array}$ & $79.16 \pm 0.10$ & $79.42 \pm 0.10$ & 69.11 \\
\hline & $\begin{array}{c}\text { trastuzumab } \\
\text { emtansine } \\
\text { (deglycosylated) }\end{array}$ & $78.63 \pm 0.10$ & $78.87 \pm 0.10$ & 68.36 \\
\hline
\end{tabular}

Supplementary Table S1: Comparison of ${ }^{\mathrm{Tw}} \mathrm{CCS}_{\mathrm{N} 2}$ values experimentally obtained by SEC-native IMMS and off-line injection of manually desalted mAb formats, for 18 therapeutic mAbs and ADCs. 

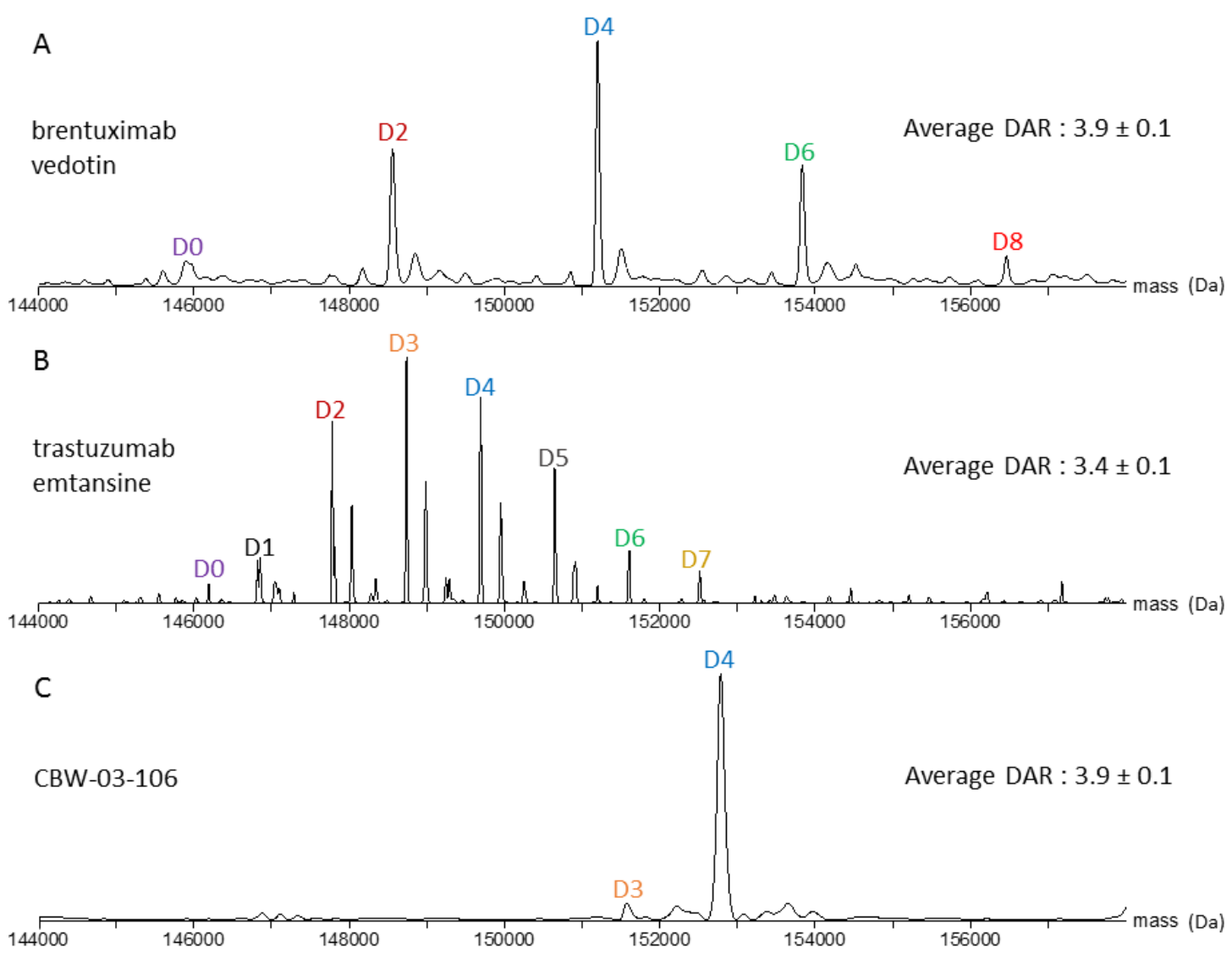

Supplementary Figure S1. Deconvoluted mass spectra of brentuximab vedotin (A), trastuzumab emtansine (B) and CBW-03-106 site specific ADC (C) obtained from online SEC-native MS.
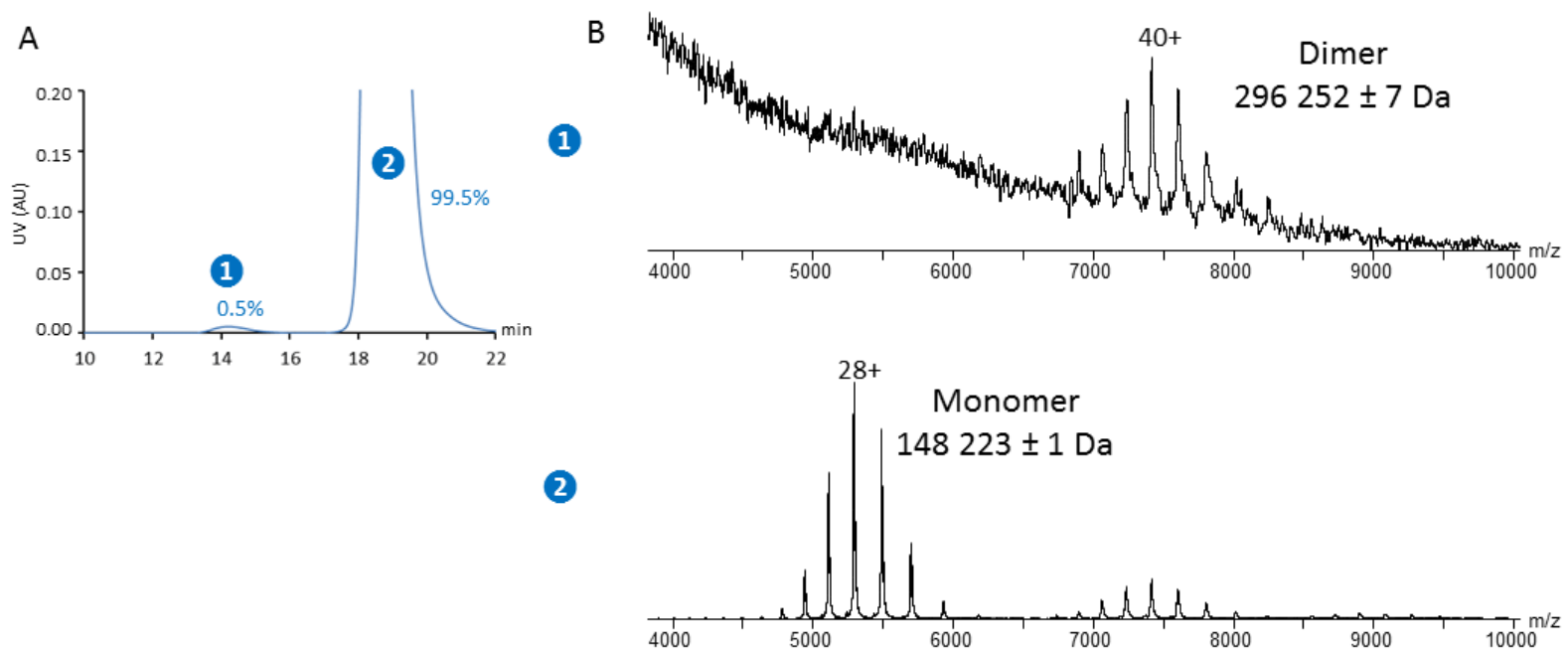
Supplementary Figure S2. Online SEC-native MS analysis of unstressed trastuzumab. (A) SEC chromatogram of unstressed trastuzumab. (B) Native mass spectra of each individual SEC peak. 